\title{
2020 Peer Reviewer Thank You
}

Network and Systems Medicine would like to thank the following individuals who reviewed manuscripts in 2020. Our journal's continued growth depends in large part on the time, effort, and expertise of its volunteer peer reviewers. On behalf of the editors, publisher, and editorial board, thank you!

\author{
Nicolas Alcaraz \\ Dominik Heider \\ Richard Röttger \\ Markus List \\ Marzieh Ayati \\ Rosa Faner \\ Dennis Görlich \\ Anne-Christin Hauschild \\ Jonathon Keeney \\ Rachel Kelly \\ Thomas Krahn \\ Manuela Lautizi \\ Weilong Li \\ Beth Peshkin \\ Sepideh Sadegh \\ Marisol Salgado \\ Michael Scherer \\ Veit Schwämmle \\ Pablo Villoslada \\ Xiao-fei Zhang \\ Alan Zuckerman
}

\author{
Ramars Amanchy \\ Giuliano Armano \\ Ankush Bansal \\ Eudes Barbosa \\ Richa Batra \\ Yana Bromberg \\ Claudio Fiocchi \\ Gihanna Galindez \\ Sascha Hauke \\ Andreas Holzinger \\ Tim Kacprowski \\ Peter Kieseberg \\ Yang-Yu Liu \\ Betram-Muller Myhsok \\ Rocio Rebollido-Rios \\ Damjana Rozman \\ Tavpritesh Sethi \\ Paola Stolfi \\ Andrew Teschendorff \\ Nina Verstraete
}

